\title{
COMPORTAMENTO MECÂNICO DO SIC ADITIVADO COM OXIDOS DE ALUMINIO E ITRIO PRODUZIDOS POR SHS*
}

\author{
Gisele Teixeira Saleiro ${ }^{1}$ \\ Danilo Correa Gonçalves ${ }^{2}$ \\ Danúbia Bordim de Carvalho ${ }^{3}$ \\ Célio Albano da Costa Neto 4 \\ José Brant de Campos 5 \\ Eduardo de Sousa Lima 6
}

\section{Resumo}

O SiC é uma cerâmica utilizada como material estrutural devido a algumas características que apresenta, tais como: alta resistência ao desgaste, ao choque térmico e a oxidação. Este composto apresenta algumas utilidades industriais do carbeto de silício, tais como selos mecânicos, vigas, rolos, chapas e placas de blindagem. Este trabalho tem como objetivo comparar as propriedades de placas hexagonais de carbeto de silício aditivado com óxidos de alumínio e ítrio comercial com placas de carbeto de silício aditivados com óxidos de alumínio e ítrio nanométricos produzidos por SHS e tratados termicamente a temperatura de $1200^{\circ} \mathrm{C}$.

Palavras-chave:SHS, politipos, dureza

\section{MECHANICAL BEHAVIOR OF SIC ADDITIVATED WITH ALUMINUM AND YTTRIUM OXIDES PRODUCED BY SHS}

\section{Abstract}

$\mathrm{SiC}$ is a ceramic used as a structural material due to some characteristics that it presents, such as: high resistance to wear, thermal shock and oxidation. This compound features some industrial uses of silicon carbide, such as mechanicals, beams, rolls, plates and shielding plates. This work aims to compare the properties of hexagonal plates of silicon carbide additives with aluminum oxides and commercial yttrium with silicon carbide plates added with aluminum oxide and yttrium oxide produced by SHS and heat treated at a temperature of $1200^{\circ} \mathrm{C}$.

Keywords: SHS, polytypes, hardness.

1 Laboratório de Materiais Cerâmicos, Seção de Engenharia Mecânica e de Materiais, Instituto Militar de Engenharia, 22291-270, Rio de Janeiro, Brasil.

2 Laboratório de Materiais Cerâmicos, Seção de Engenharia Mecânica e de Materiais, Instituto Militar de Engenharia, 22291-270, Rio de Janeiro, Brasil.

3 Laboratório de Materiais Cerâmicos, Seção de Engenharia Mecânica e de Materiais, Instituto Militar de Engenharia, 22291-270, Rio de Janeiro, Brasil.

4 Departamento de Engenharia Metalúrgica e de Materiais, Universidade Federal do Rio de Janeiro, 21941-901, Rio de Janeiro, Brasil

5 Departamento de Engenharia Mecânica, Universidade do Estado do Rio de Janeiro, 20550-900, Rio de Janeiro, Brasil

6 Laboratório de Materiais Cerâmicos, Seção de Engenharia Mecânica e de Materiais, Instituto Militar de Engenharia, 22291-270, Rio de Janeiro, Brasil. 


\section{INTRODUÇÃO}

O carbeto de silício é considerado uma cerâmica avançada, pois é uma cerâmica de alta tecnologia que vêm sendo aplicadas nas áreas aeroespacial, eletrônica, nuclear e em muitas outras que passaram a exigir materiais com qualidade excepcionalmente elevada [1].

Em relação à cristalografia, o SiC é um dos exemplos mais conhecidos de politipismo, fenômeno em que um material pode adotar diferentes estruturas cristalinas, que variam em uma dimensão, ou seja a mudança ocorre na seqüência dos empilhamentos dos planos e não existe variação na sua composição química. $O$ carbeto de silício se cristaliza em mais de 200 politipos.

Os sistemas desses politipos são: cúbica, representada pela letra $C$, hexagonal, identificada pela letra $\mathrm{H}$ e romboédrica indicada pela letra $\mathrm{R}$ [2]. A figura 1 demonstra alguns polítipos do SiC.

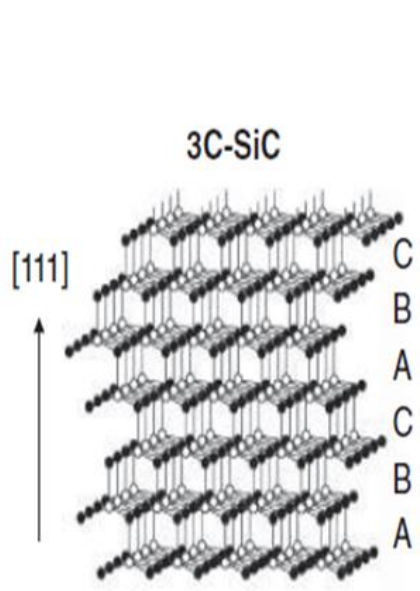

(a)

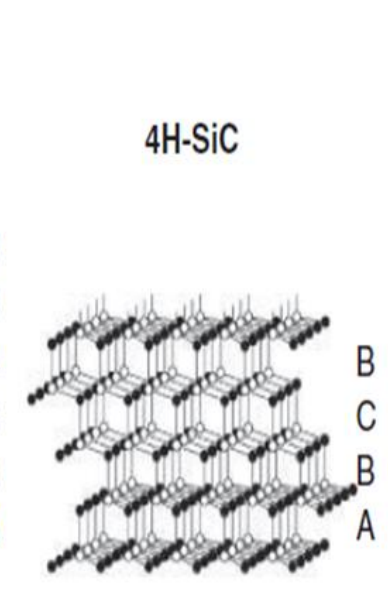

(b)

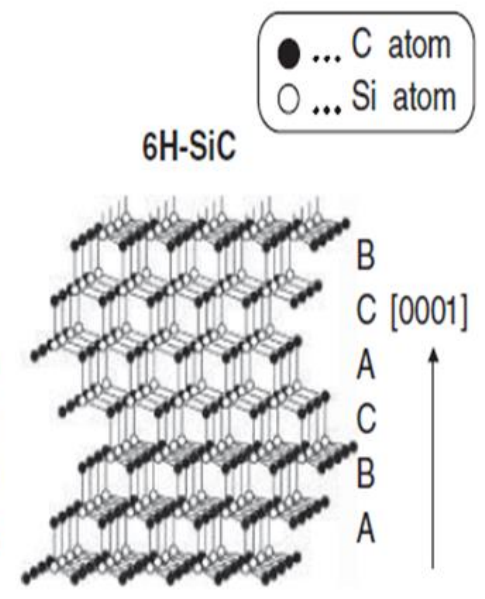

(c)

FIG 1 Estruturas do $\mathrm{SiC}$ (a) 3C-SiC, (b) 4H-SiC, e (c), 6H-SiC [3]

O pó de carbeto de silício pode ser produzido de várias formas: redução carbotérmica, síntese por fase gasosa e SHS. A primeira é a mais importante sendo conhecida como processo Acheson. Este método ocorre pela redução carbotérmica da sílica. O processo utiliza um forno de alta resistência. Neste método é misturada uma fonte de sílica e uma fonte de carbono que são colocados até a altura dos eletrodos. A fonte de carbono pode ser coque de petróleo ou carvão. A mistura é aquecida através da corrente elétrica transmitida pelos eletrodos que estão localizados nas extremidades do forno [4] [5]. O carbeto de silício possui rotas de processamento e sinterização diferentes. E importante ressaltar que a microestrutura gerada e as características do material serão diferentes, dependendo do processo que foi empregado.

No processo de sinterização do $\mathrm{SiC}$, a temperatura de $2000^{\circ} \mathrm{C}$ ocorre a transformação de fase do $\mathrm{SiC}$. A forma $\alpha$-SiC ocorre em temperaturas mais altas. $\mathrm{Na}$ faixa de temperatura de $2000{ }^{\circ} \mathrm{C}$ ocorre uma transformação cristalográfica da fase $\beta$ $\rightarrow$ na fase $\alpha$. Com isso, a fase $\beta$-SiC pode ser caracterizada como uma fase metaestável termicamente [6]. 
Essa transformação ocorre pelo mecanismo de maclagem, no qual existe uma simetria em espelho da rede cristalina. Essa transformação de fase ocorre por meio de impurezas [2]. A sinterização sem presença de pressão só ocorre caso haja a inserção de aditivos. Portanto, muitos óxidos metálicos têm sido estudados com esta finalidade a fim de diminuir a temperatura de sinterização. Os aditivos de sinterização de óxidos de metais relatados até agora incluem $\mathrm{Al}_{2} \mathrm{O}_{3}, \mathrm{MgO}, \mathrm{Lu}_{2} \mathrm{O}_{3}$, $\mathrm{Y}_{2} \mathrm{O}_{3}, \mathrm{Al}_{2} \mathrm{O}_{3}-\mathrm{Y}_{2} \mathrm{O}_{3}-\mathrm{MgO}, \mathrm{Al}_{2} \mathrm{O}_{3}-\mathrm{YAG}, \mathrm{Al}_{2} \mathrm{O}_{3}-\mathrm{Y}_{2} \mathrm{O}_{3}-\mathrm{CaO}, \mathrm{Al}_{2} \mathrm{O}_{3}-\mathrm{Y}_{2} \mathrm{O}_{3}-\mathrm{SiO}_{2}, \mathrm{Al}_{2} \mathrm{O}_{3}-$ $\mathrm{Y}_{2} \mathrm{O}_{3}$ mais utilizado e o sistema de vidro Y-Mg- Si-Al-O-N. Estes óxidos diminuem a temperatura de sinterização do $\mathrm{SiC}$ para $1700-1850{ }^{\circ} \mathrm{C}$ [7] [8].Os primeiros estudos do sistema $\mathrm{Al}_{2} \mathrm{O}_{3}-\mathrm{Y}_{2} \mathrm{O}_{3}$ foram feitos por Warshaw em1959, estabelecendo a formação do eutético estável $\mathrm{Al}_{2} \mathrm{O}_{3}$-YAG a $1826^{\circ} \mathrm{C}$ (FIG.2). O YAG apresenta boa estabilidade mecânica e química sendo aplicado em processos que envolvem energia e temperaturas altas. De acordo com o diagrama sua composição em porcentagem de $\mathrm{Y}_{2} \mathrm{O}_{3}$ é de aproximadamente 37,5\%. O YAP de acordo com 0 diagrama possui porcentagem de Ítria de 50\%em mols e temperatura de estabilização de $1702^{\circ} \mathrm{C}$, sendo maior do que a do composto YAG. A formação metaestável desta fase é demonstrada através da linha mais clara formando o compósito $\mathrm{Al}_{2} \mathrm{O}_{3}-\mathrm{YAP}$. O YAM apresenta $67 \%$ em mols de Ítria, sendo considerada uma fase intermediária [9][10].

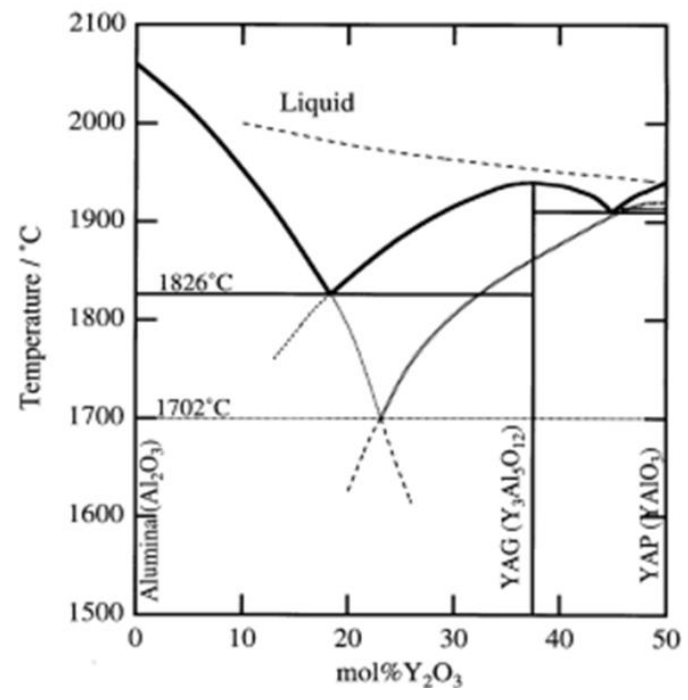

FIG 2 Diagrama de Fases [9]

A mistura de $\mathrm{Al}_{2} \mathrm{O}_{3}$ e $\mathrm{Y}_{2} \mathrm{O}_{3}$ e do compósito $\mathrm{Al}_{2} \mathrm{O}_{3}$-YAG foram usados nas proporções de 5, 10 e 15\% em peso. As temperaturas de sinterização utilizadas neste estudo foram de 1800,1850 e $1900^{\circ} \mathrm{C}$. De acordo com o autor as propriedades do material sinterizado foram alcançadas, pois foram encontrados valores semelhantes ao proposto na literatura, tais como baixa porosidade aparente e dureza no intervalo de 20 a $25 \mathrm{GPa}$ [7].

\section{SHS}

A síntese por combustão auto sustentada à alta temperatura, SHS ("Self Propagation High Temperature Synthesis") é um método de combustão homogênea, no qual ocorre a propagação da onda pela mistura heterogênea [11]. Este método tem por objetivo a produção de nanopartículas através do aquecimento via reações 
exotérmicas. O método consiste em aquecer um pó compactado em um forno até que a reação de combustão ocorra em toda a amostra. É importante destacar que durante o processo ocorre grande liberação de gases [12]. As reações do método SHS podem ser classificadas de acordo com o estado físico do reagente na máxima temperatura que o sistema pode atingir, chamada de temperatura adiabática. São elas: reação sólido-sólido, reação sólido-líquido, reação líquido-líquido e reação sólido (líquido)-gás, sendo a mais comum areação sólido-sólido [7].

Este trabalho tem como objetivo comparar as propriedades de placas hexagonais de carbeto de silício aditivado com óxidos de alumínio e ítrio comercial com placas de carbeto de silício aditivados com óxidos de alumínio e ítrio nanométricos produzidos por SHS e tratados termicamente a temperatura de $1200^{\circ} \mathrm{C}$.

\section{MATERIAIS E MÉTODOS}

Foram preparados dois grupos de amostras diferentes. O primeiro grupo de amostras consiste na mistura do pó de Carbeto de Silício com óxidos de Alumínio e Ítrio comerciais ( $\mathrm{SiC} 1$ ). No segundo grupo foi realizada a mistura do pó de Carbeto de Silício com aditivos produzidos por SHS na máxima cristalinidade mantendo a escala nanométrica. A máxima cristalinidade foi obtida com tratamento térmico a $1200^{\circ} \mathrm{C}$ (SiC 2).O método utilizado para a preparação do pó nanométrico foi à síntese por combustão homogênea auto sustentada (SHS). A proporção quantitativa de nitratos estipulada está relacionada com o diagrama de fases que corresponde a $81,5 \%$ em mols de $\mathrm{Al}_{2} \mathrm{O}_{3}$ e $18,5 \%$ em mols de $\mathrm{Y}_{2} \mathrm{O}_{3}$. A reação foi feita para uma solução de 0,1 mols. Os nitratos foram diluídos em água deionizada e a mistura homogeneizada. Após a dissolução, a solução foi colocada no Agitador Magnético com Aquecimento IKA RCT SAFETYCONTROL, previamente ligado a uma temperatura fixa de $150^{\circ} \mathrm{C}$. O processo termina quando pó é produzido e a chama cessa. A amostra recebeu tratamento térmico de $1200^{\circ} \mathrm{C}$. A taxa utilizada foi de $3^{\circ} \mathrm{C} / \mathrm{min}$ e o patamar de queima de $3 \mathrm{~h}$ no forno JUNG no Instituto Militar de Engenharia. $1200^{\circ} \mathrm{C}$.

Os pós foram tratados antes da mistura utilizando grall e pistilo para a separação dos grãos e posteriormente passaram pelo agitador de peneiras marca +GF+. A proporção de aditivo foi de $5 \%$ em peso de material sendo a água utilizada com solvente [13].

Os corpos foram prensados utilizando-se uma matriz hexagonal de $37 \mathrm{~mm}$ de lado. A matriz foi lubrificada com estearina antes da colocação dos pós. Utilizou-se cerca de $60 \mathrm{~g}$ de pó para cada produção de cada peça. A prensagem uniaxial (FIG 3), foi realizada no Centro de Tecnologia Aeronáutica com o auxílio da prensa hidráulica EKA com capacidade de até40 t. A carga utilizada no processo foi de 20 t (55 MPa) pelo tempo de $1 \mathrm{~min}[14]$. 


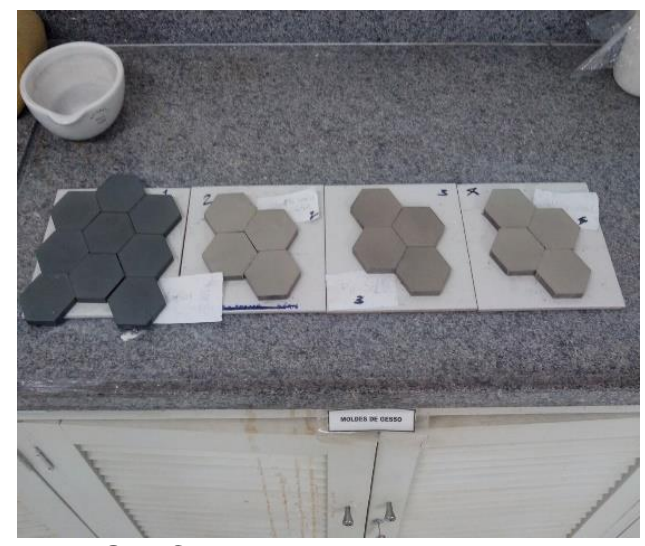

FIG 3 Corpos Verdes Hexagonais

Após a prensagem uniaxial os corpos de prova foram sinterizados na temperatura de $1950^{\circ} \mathrm{C}$ com patamar de 30 minutos. As amostras sofreram a limpeza da superfície para a realização de algumas medidas, tais como: contração, perda de massa, densidade, caracterização microestrutural e determinação das propriedades mecânicas.

As propriedades mecânicas foram verificadas através dos cálculos de densificação e porosidade de acordo com a norma ABNT NBR 6220:2011.

Após a verificação da porosidade as amostras foram submetidas ao ensaio de raios -X e análise de Rietveld e ensaio de dureza Vickers.

A análise de Rietveld teve como objetivo verificar os materiais, bem como os seus percentuais após a sinterização. Para a determinação de retração linear dos corposde- prova após queima foi utilizado um paquímetro digital com precisão de 0,01 $\mathrm{mm}$ para a medição dos comprimentos inicial e final.

A determinação da densidade e porosidade aparente foram realizadas com base nas normas ASTM C20 e ABNT NBR 6220. Para o processo utilizou-se abalança analítica Gehaka BK 300. As amostras foram colocadas em água em um béquer de $1 \mathrm{~L}$, e fervidas por $2 \mathrm{~h}$. Após as amostras atingirem a temperatura ambiente mediu-se a massa imersa $(\mathrm{m}$ i) e posteriormente a medição da massa saturada $(\mathrm{m} \mathrm{u})$. As amostras foram colocadas em estufa por $12 \mathrm{~h}$ a temperatura de $80^{\circ} \mathrm{C}$ para medida da massa seca $(\mathrm{m} \mathrm{s})$.

O cálculo da massa específica aparente (MEA) foi obtido a partir da razão entre a massa do corpo-de- prova seco e seu volume ou ainda a razão entre amassa seca e a diferença entre a massa saturada e a massa do corpo-de-prova imerso em água.

O ensaio de dureza foi baseado na norma ASTM C 1327-03 (American Standard for Testing Materials), utilizando as medidas de indentação Vickers. O penetrador é uma pirâmide de diamante de base quadrada e com um ângulo de $136^{\circ}$ entre faces opostas, equipamento Shimatzu XX do IME, carga HV 0,3por 15 segundos. É importante ressaltar que este ensaio é especialmente para materiais muito duros, ou corpos de prova muito finos, pequenos e irregulares, sendo por isso conhecido como ensaio universal.

\section{RESULTADOS E DISCUSSÃO}

As figuras 4 e 5 apresentam os difratogramas dos corpos de prova representativos das amostras aditivadas com pó comercial e das amostras aditivadas com pó resultante da reação do SHS. 


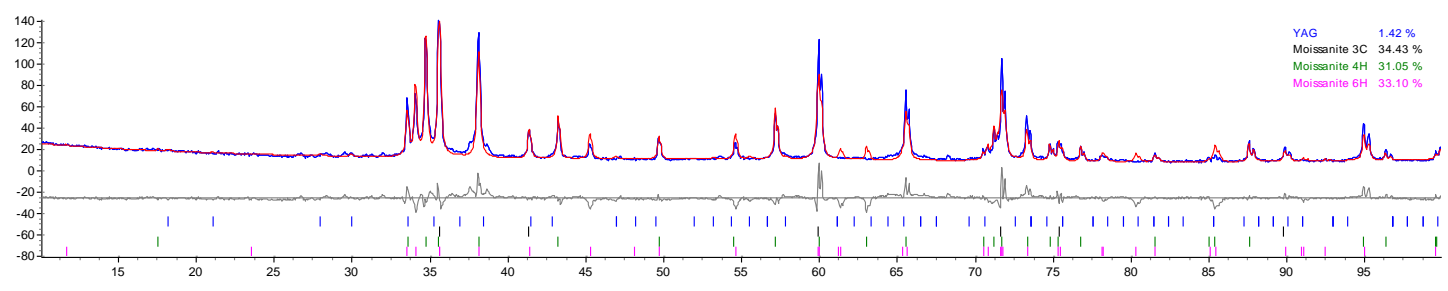

Figura 4.Análise de Rietveld das peças aditivadas com pó comercial

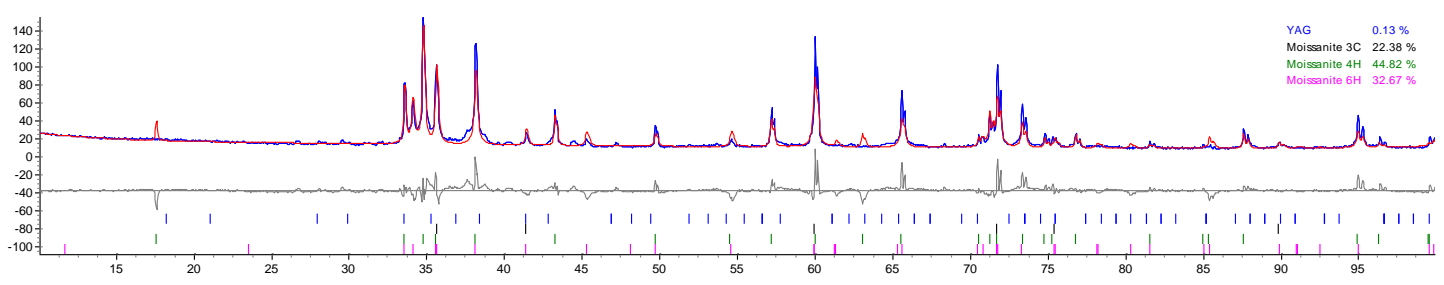

Figura 5.Análise de Rietveld das peças aditivadas com pó SHS

Pode-se observar que em ambos os casos foram encontradas as mesmas fases, como por exemplo, o YAG, fase formada a $1826^{\circ} \mathrm{C}$ no ponto eutético do diagrama de fases do sistema $\mathrm{Al}_{2} \mathrm{O}_{3}-\mathrm{Y}_{2} \mathrm{O}_{3}$. De acordo com o difratograma foi possível observar que houve que também houve a transformação da fase $\beta \rightarrow$ na fase $\alpha$ conforme relatado na literatura [6].

A tabela 1 apresenta os resultados das propriedades dos corpos de prova aditivados com óxidos de alumínio e ítrio comerciais e corpos de prova aditivados com óxidos de alumínio e ítrio obtidos pela técnica de SHS.

Tabela 1.Propriedades do corpo de prova

\begin{tabular}{lccccc}
$\begin{array}{l}\text { Tipo de } \\
\text { aditivo }\end{array}$ & $\begin{array}{c}\text { Densificação } \\
\text { (\%) }\end{array}$ & MEA & $\begin{array}{l}\text { Retração } \\
\text { Linear }\end{array}$ & $\begin{array}{l}\text { Porosidade } \\
\text { Aparente }\end{array}$ & $\begin{array}{l}\text { Dureza } \\
\text { Vickers } \\
\text { (GPa) }\end{array}$ \\
\hline SIC 1 & $90,9 \pm 0,94$ & $2,93 \pm$ & $53,2 \pm 0,08$ & $4,30 \pm 1,30$ & $\begin{array}{l}20,9 \\
\pm 1,4\end{array}$ \\
& & 0,03 & & & \\
\hline SIC 2 & $91,4 \pm 0,49$ & $2,91 \pm$ & $51,8 \pm 0,99$ & $1,08 \pm 0,12$ & 28,9 \\
& & 0,02 & & & $\pm 1,3$ \\
\hline
\end{tabular}

Apesar das amostras aditivadas com pó comercial apresentarem resultados muito próximos de densificação comparado as amostras aditivadas com pós produzidos por SHS, os resultados de dureza foram melhores nas peças aditivadas com pó de SHS. Este resultado demonstra a influência da porosidade nas propriedades mecânicas dos materiais cerâmicos. Comparado a outros trabalhos anteriores utilizando o carbeto de silício aditivado com alumina ítria, pode-se citar o trabalho apresentado por Lima [7], no qual os valores encontrados para dureza para óxidos do sistema alumina ítria estão na faixa de 20 à $25 \mathrm{GPa}$. O valor apresentado na mistura SiC 2 foi maior do que o encontrado em pesquisas anteriores, incluindo outros sistemas e materiais.

Dias também utilizou a dureza Vickers em seu trabalho para placas de Carbeto de Tungstênio utilizando de 5 à 15\% de volume de Cobalto como aditivo. Neste trabalho o maior valor atingido de dureza foi de 16,09 GPa [15].Santos [16] utilizou o sistema 
$\mathrm{Al}_{2} \mathrm{O}_{3} \mathrm{Nb}_{2} \mathrm{O}_{5}$ aditivado com LiF com o objetivo de blindagem balística. Os valores maiores valores de dureza foram na ordem de 13,04 GPa quando adicionados 0,5\% de aditivo.

A tabela 2 apresenta os valores de dureza Vickers para alguns sistemas.

Tabela 2.Dureza de Cerâmicas Avançadas [17]

\begin{tabular}{lccccc}
\hline $\begin{array}{l}\text { Propriedad } \\
\text { e }\end{array}$ & SIC & $\mathrm{Si}_{3} \mathrm{~N}_{4}$ & $\mathrm{ZrO}_{2}$ & $\mathrm{Al}_{2} \mathrm{O}_{3}$ & AIN \\
\hline $\begin{array}{l}\text { Dureza } \\
\begin{array}{l}\text { Vickers } \\
\text { (GPa) }\end{array}\end{array}$ & $20-30$ & $8-19$ & $10-15$ & $18-23$ & $8-13$ \\
\hline
\end{tabular}

As figuras 6 e 7 apresentam a microscopia eletrônica de varredura por elétrons retroespalhados das amostras SiC $1 \mathrm{E} \mathrm{SiC} 2$.

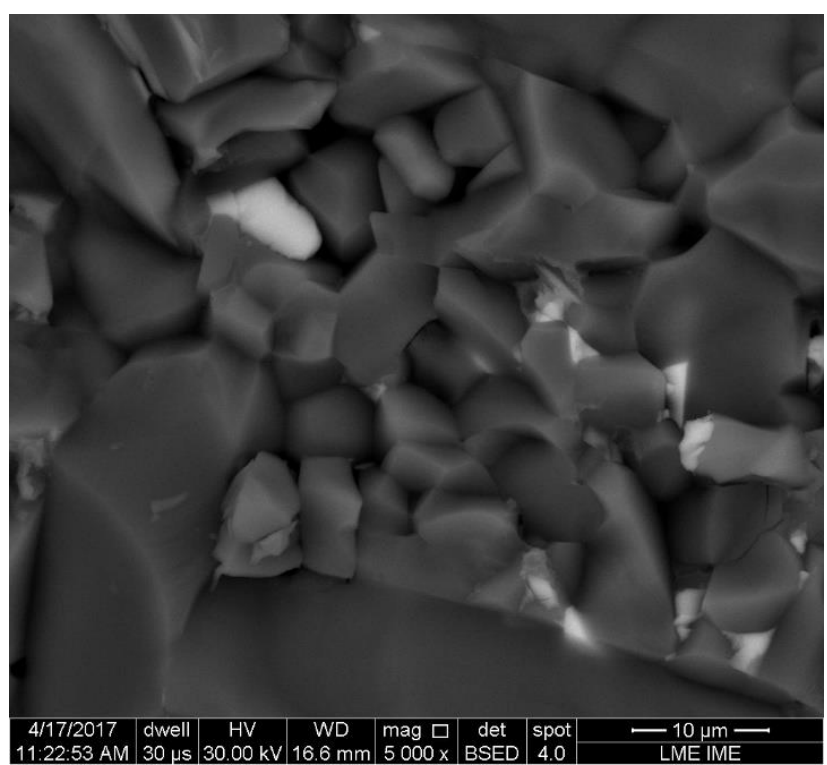

Figura 6: MEV amostra SiC 1 


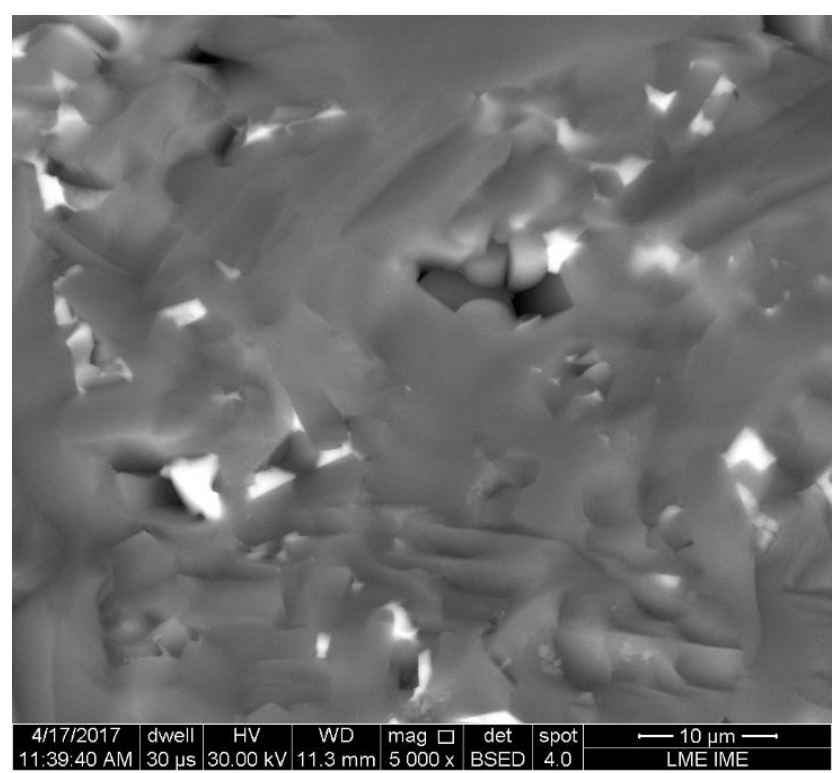

Figura 7: MEV amostra SiC 2

É possível observar nas figuras 6 e 7 pequenas regiões com presença de molhamento heterogêneo. De acordo com a área observada no MEV foi detectado que há maior dispersão de molhamento nas amostras SiC 2.

\section{CONCLUSÃO}

É possível observar que em ambas as peças houve a formação YAG, fase mais estável do diagrama de fases do sistema alumina- ítria.

Foi possível observar a transformação da fase $\beta \rightarrow$ na fase $\alpha$ conforme relatado na literatura.

De acordo com os resultados foi possível observar que as peças aditivadas com pós provenientes da reação SHS apresentaram valores menores e significativos de porosidade aberta refletindo em melhores propriedades mecânicas quando comparadas as peças aditivadas com pós comerciais.

\section{REFERÊNCIAS}

[1] Associação Brasileira de Cerâmica

Disponível em: http://abceram.org.br/definicao-e- classificação/

Acesso em: 04/05/2016

[2] CASADY, J. B., JOHNSON, R. W., Status of Silicon Carbide (SiC) as a Wide-Band gap Semiconductor for High-Temperature Applications: a Review, Solid-State Electronics, v. 39, n. 10, p. 1409-22, 1996.

[3] KIMOTO, T., COOPER, J. A., Fundamentals of Silicon Carbide Technology, Wiley, 2014.

[4] GUPTA, G. S., Heat-Transfer model of the Acheson Process, Metallurgical and MaterialsTransaction, v. 32, p. 1301-1308, 2001. 
[5] NASSAU, KURT. Synthetic Moissanite: a new man-madejewel. Current Science, v. 79,n. 11, Dec. 2000.

[6] WACHTMAN, J. B., Structural Ceramics, Academic Press Inc, 1989.

[7] LIMA, E. S., Sinterização do SiC com Adição do Compósito Al2O3-YAG.

Tese (Doutorado em Ciência dos Materiais) - IME, 2006.

[8] NOVIYANTO, A. YOON, D. H., One Component Metal Oxide Sintering Additive for $\beta$-SiC Based on Thermodynamic Calculation and Experimental Observations, Met. Mater. Int., Vol. 18, N o. 1 p. 63-68, 2012.

[9] YASUDA, H., OHNAKA, I., MIZUTANI, Y. and WAKU, Y., Selection of Eutectic Systems in Al2O3-Y2O3 Ceramics, Science and Techn. Of Adv. Mater., v. 2, n. 1, p. 67-71, 2001.

[10] LI, W. Q., GAO, L., Processing, Microstructure and Mechanical Properties of 25 vol\% YAG-Al2O3 Nanocomposites, NanoStructured Materials, v. 11, n.8, p. 1073-80, 1999.

[11] ROGACHEV, A. S.; BARAS, F. Models of SHS: An Overview, International Journal of Self-Propagating High-Temperature Synthesis, 2007, Vol. 16,No. 3, pp. 141-153.

[12] KINGSLEY, J.J.; PEDERSON, L.R. Energetic Materials in Ceramics Synthesis, MRS Proceedings, 296, (1992).

[13] CORREA, D., SALEIRO, G.T,et al. Microstructural Characterization and Influence of Ceramography at Microhardness Test of Silicon Carbide, Materials Research, 2017.

[14] ALVES, J. T; ASSIS, J. M. T.; MELO, F. C. L.; LOPES, C. M. A. Produção e caracterização de cerâmicas para blindagem balística. Congresso Brasileiro de Engenharia e Ciência dos Materiais, 2010.

[15] DIAS, A.M.S. Avaliação da tenacidade à fratura através do ensaio de indentação em pastilhas de metal duro, Revista Matéria, v. 14, n. 2, pp. 869-877, 2009.

[16] SANTOS, J.L. Comportamento Balístico de Componente Cerâmico a Base de Al2O3Nb2O5-LiF em blindagem (Tese de Doutorado- IME), 2016.

[17] CUTLER, R. A.: High-Toughness Silicon Carbide as Armor, J. Am. Ceram.Soc, v. 8, p. 2217-2226. 2005. 
WACHTMAN, J. B., Structural Ceramics, Academic Press Inc., 1989. 